\title{
Efficient test compaction for combinational circuits based on Fault detection count-directed clustering
}

\author{
A. El-Maleh and S. Khursheed
}

\begin{abstract}
Test compaction is an effective technique for reducing test data volume and test application time. The authors present a new static test compaction technique based on test vector decomposition and clustering. Test vectors are decomposed and clustered for faults in an increasing order of faults detection count. This clustering order gives more degree of freedom and results in better compaction. Experimental results demonstrate the effectiveness of the proposed approach in achieving higher compaction in a much more efficient CPU time than the previous clustering-based test compaction approaches.
\end{abstract}

\section{Introduction}

Recent advances in very large scale integration (VLSI) technology have enabled the fabrication of systems-on-a-chip with millions of transistors. This tremendous increase in transistor count has resulted in large increase in test data volume that often exceeds current testers' memory capacity. In order to reduce the test data volume, two basic strategies have been investigated. The first strategy is based on reducing the required number of test vectors needed to achieve a given desired fault coverage, known as test compaction. The second strategy is based on representing the test data in a compressed form in the tester and using decompression circuitry on chip to decompress the test data before application, known as test compression. Both strategies are necessary to reduce test data volume and test application time.

Test compaction techniques can be classified as static or dynamic. In static test compaction, the number of test vectors is reduced after they are generated, whereas in dynamic test compaction, the number of test vectors is minimised during the automatic test pattern generation (ATPG) process. Static test compaction algorithms for combinational circuits can be divided into three broad categories [1]: (1) redundant vector elimination, (2) test vector modification; and (3) test vector addition and removal. In the first category, compaction is performed by dropping redundant test vectors. A redundant test vector is a vector whose faults are all detectable by other test vectors. Static compaction algorithms falling under this category are either based on set covering [2-4] or test vector reordering and fault simulation [5-9]. In the second category, compaction is performed by modifying test vectors. This is achieved by merging of compatible test vectors based on test relaxation or raising [10-13], essential fault pruning [7, 13-15], or test vector decomposition and clustering [1, 16, 17].

(C) The Institution of Engineering and Technology 2007

doi:10.1049/iet-cdt:20070004

Paper first received 6th January 2006 and in revised form 6th February 2007

A. El-Maleh is with the Department of Computer Engineering, King Fahd University of Petroleum and Minerals, P.O. Box 1063, Dhahran 31261, Saudi Arabia

S. Khursheed is with the Department of Electronics and Computer Science, University of Southampton

E-mail: aimane@ccse.kfupm.edu.sa
Finally, the third category of static compaction algorithms consists of compaction algorithms that add new test vectors to a given test set in order to remove some of the already existing test vectors $[8,18]$.

Static compaction techniques are preferred to dynamic compaction for several reasons. First, generating compact test sets using dynamic compaction is more time-consuming as many attempts to modify partially specified test vectors to detect additional faults often fail [12]. In addition, dynamic compaction does not take advantage of random test generation that makes the ATPG process more efficient. Second, static compaction is ATPG-independent allowing test sets to be generated using more efficient ATPG techniques. Finally, static compaction techniques could result in more compact test sets than dynamic compaction techniques as indicated by the results in $[9,15]$.

Recently, two static compaction techniques based on test vector decomposition and clustering have been proposed in $[1,16,17]$. The first technique, called independent fault clustering (IFC) [1, 16], is based on clustering test vectors according to independent fault sets. The second technique, called class-based clustering (CBC) [1, 17], is based on classifying test vectors into classes and then eliminating test vectors by moving their components to other test vectors.

In this work, we propose a new test compaction technique based on test vector decomposition and clustering. Test vector decomposition and clustering is performed for faults based on the number of test vectors detecting each fault, that is fault detection count. This is in contrast to IFC which clusters test vectors based on independent fault sets.

\section{Proposed test compaction technique}

Test vector decomposition is the process of decomposing a test vector into its atomic components. An atomic component is a child test vector that is generated by relaxing its parent test vector for a single fault $f$. That is, the child test vector contains only the assignments necessary for the detection of $f$. Besides, the child test vector may detect other faults in addition to $f$.

In IFC $[1,16]$, independent fault sets (IFSs) with respect to a given test set are first derived. Two faults are independent if they are not detected by the same test vector. Then, test vector clustering is performed based on the derived IFSs. This is motivated by the fact that the size of the largest IFS 
gives an upper bound on the possible size of the final test set after compaction and that test vector components for faults belonging to different IFSs are potentially compatible. During test vector clustering, compatible components, corresponding to compatible faults, are mapped to the same compatibility set. Whenever a component is mapped to a compatibility set, it is merged with the partial test vector of that compatibility set. At the end of the clustering process, each compatibility set represents a single test vector.

Our proposed test compaction technique, fault-detection count-based clustering (FCC), is based on clustering test vector components based on fault-detection count. Components derived from faults with the smallest detection count are clustered first followed by faults with increasing detection count. This is motivated by the fact that faults with $N$ detection count have $\boldsymbol{N}$ test vector components and have a higher chance of being compatible with the existing clusters. If a test vector component is not compatible with all the existing clusters, other test vector components are attempted. A new cluster is created only when all the $\boldsymbol{N}$ test vector components are not compatible with all the existing clusters.

The FCC algorithm is shown in Fig. 1 and proceeds as follows. First, the given test set $T$ is fault-simulated without fault dropping. This step is performed to find the

\section{Algorithm FCC(T)}

1. Fault simulate $T$ without fault dropping.

1.1. Record the number of test vectors detecting each fault.

2. Group the faults by their detection count.

2.1. Sort the faults in ascending order of their detection count.

3. For every essential fault $f$ that is detected by a test vector $t$ :

3.1. Extract the atomic component $c_{f}$ from $t$.

3.2. If the number of compatibility sets is zero, create a new compatibility set, map $c_{f}$ to it, and then go to Step 3.

3.3. Map $\mathcal{c}_{f}$ to an existing compatibility set, if possible, and then go to Step 3 .

3.4. Create a new compatibility set and map $c_{f}$ to it.

4. Fault simulate all the compatibility sets and drop all the remaining (Non-Essential) faults that are detected.

5. For the remaining non-essential (un-detected) fault(s) $f$ that is detected by a set of test vector $T^{\prime}$ :

5.1. For every test vector $t^{\prime}$, where $t^{\prime}$ is a member of $T^{\prime}$ :

5.2. Extract the atomic component $c_{f}$ from $t$ '.

5.3. If the number of compatibility sets is zero, create a new compatibility set, map $c_{f}$ to it, and then go to Step 5.

5.4. Map $c_{f}$ to an existing compatibility set, if possible, and then go to Step 5, otherwise go to Step 5.1.

6. Random fill test vectors of all the compatibility sets.

7. Fault simulate all the compatibility sets and drop all the remaining (Non-Essential) faults that are detected.

8. For the remaining non-essential (un-detected) fault(s) $f$ that is detected by a set of test vector $T$ ':

8.1. For every test vector $t^{\prime}$, where $t^{\prime}$ is a member of $T^{\prime}$ '

8.2. Extract the atomic component $\mathcal{c}_{f}$ from $t$.

8.3. Map $c_{f}$ to an existing compatibility set, if possible, and then go to Step 8, otherwise go to Step 8.1.

8.4. Create a new compatibility set and $\operatorname{map} \mathcal{c}_{f}$ to it.

9. Random fill all the vectors of $T^{*}$.

10. Return $T^{*}$.

Fig. 1 Fault-detection count-based clustering (FCC) number and set of test vectors that detect every fault. Second, all the faults are sorted using their detection count in ascending order. Next, test vector components for essential faults (i.e. detection count $=1$ ) are clustered. In this step, for every essential fault $f$ detected by $t$, the atomic component $\mathrm{c}_{f}$ corresponding to $f$ is extracted from $t$. Then, for every compatibility set $\mathbf{C S}_{i}$, if $\mathrm{c}_{f}$ is compatible with the partial test vector in $\mathbf{C S}_{i}, \mathrm{c}_{f}$ is mapped to $\mathbf{C S}_{i}$. On the other hand, if the number of compatibility sets is zero or $\mathrm{c}_{f}$ is incompatible with all partial test vectors in the existing compatibility sets, a new compatibility set is created and $\mathbf{c}_{f}$ is mapped to it.

Next, the algorithm fault simulates the existing compatibility sets and drops all detected faults. This step saves the computation time which is otherwise spent on extracting atomic components of yet unmapped, non-essential faults and then either mapping them to the existing compatibility sets or creating a new compatibility set for such faults. In addition, it could result in higher compaction.

The algorithm then focuses on the remaining unmapped, non-essential faults. This step exhaustively checks every component of a non-essential fault and attempts to minimise creating a new compatibility set. For every fault, an atomic component of a fault $f$ is extracted. If it is incompatible with all partial test vectors in the existing compatibility sets, a new component is tried. In this step, a new compatibility set is created only if the number of compatibility sets is zero, which is possible only when there are no essential faults. At this point, only those non-essential faults remain which require a new compatibility set and none of their atomic component could be mapped to any of the partially filled existing compatibility sets.

The algorithm then randomly fills the partially filled test vectors of the existing compatibility sets and fault-simulates all the compatibility sets. This is done to maximise the chances of detecting yet unmapped, non-essential faults and therefore save an extra compatibility set. It should be noted that random filling in step 6 does not affect compaction, since it is guaranteed that none of the remaining test vector components could map to any of the existing partially filled test vectors.

Finally, the unmapped remaining non-essential faults are clustered. The algorithm creates an additional compatibility set for the remaining non-essential faults only if all components of a fault $f$ are incompatible with all partial test vectors in the existing compatibility sets. It should be noted that at this stage compatibility is only checked with newly created clusters. At the end, the algorithm randomly fills the remaining partially filled test vectors and returns the compatibility sets as the compacted test set.

It is worth mentioning that for large circuits with large number of faults, fault-simulation without dropping can be restricted to a $k$ number of fault detects. The value of $k$ chosen provides a tradeoff in memory and CPU time requirement and the achieved level of compaction.

We next illustrate the steps of the proposed FCC algorithm through an example. Table 1 shows a set of four test vectors along with their detected faults and the components generated for each fault. Faults $f_{1}$ and $f_{2}$ are essential faults and will be clustered first resulting in two sets as shown in Table 2. We assume in this example that fault-simulating the resulting compatibility sets will not detect additional faults. Then, faults with detection count $=2$ will be clustered next, i.e., faults $f_{3}, f_{4}, f_{5}, f_{7}$ and $f_{8}$. The first component of $f_{3}=\mathrm{x} 0 \times \mathrm{x} \times \mathrm{x} 11 \mathrm{x} 1$ will be attempted for clustering and it will be found incompatible with the existing sets. The second component of $f_{3}=\mathrm{x} 0 \times 1 \times 1 \times x \times 1$ is then successfully clustered into the second set. The first component of 
Table 1: Example test vectors and their components

\begin{tabular}{|c|c|c|c|}
\hline \multicolumn{2}{|c|}{ Test Vector } & \multirow{2}{*}{$\begin{array}{l}\begin{array}{l}\text { Fault } \\
\text { detected }\end{array} \\
f_{1}\end{array}$} & \multirow{2}{*}{$\begin{array}{l}\begin{array}{l}\text { Fault } \\
\text { component }\end{array} \\
0 \times 0 \times x 1 \times \times x 0\end{array}$} \\
\hline$v_{1}$ & 0000111110 & & \\
\hline & & $f_{4}$ & $00 x \times 1 \times x 1 x x$ \\
\hline \multirow[t]{4}{*}{$v_{2}$} & 1101101001 & $f_{2}$ & $1 \times x 1 \times \times 10 \times 1$ \\
\hline & & $f_{5}$ & $x x x \times 10 x 0 x x$ \\
\hline & & $f_{6}$ & $1 \times \times 1 \times 0 \times 0 \times 1$ \\
\hline & & $f_{8}$ & $x 1 x \times 1 \times x 001$ \\
\hline \multirow[t]{4}{*}{$v_{3}$} & 1010111101 & $f_{3}$ & $x 0 x x x \times 11 x 1$ \\
\hline & & $f_{5}$ & $x \times x \times 1 \times x 101$ \\
\hline & & $f_{6}$ & $1 \times x \times 11 \times 10 x$ \\
\hline & & $f_{7}$ & $1 \times 1 \times 1 \times x 10 x$ \\
\hline \multirow[t]{5}{*}{$v_{4}$} & 0011110101 & $f_{3}$ & $x 0 x 1 \times 1 \times x \times 1$ \\
\hline & & $f_{4}$ & $00 \times x \times 1 \times 1 \times 1$ \\
\hline & & $f_{6}$ & $00 x \times 11 \times x 0 x$ \\
\hline & & $f_{7}$ & $x \times 1 \times 1 \times 0 x 0 x$ \\
\hline & & $f_{8}$ & $x \times x 11 \times 0101$ \\
\hline
\end{tabular}

$f_{4}=00 \mathrm{xx} 1 \mathrm{x} \times 1 \times \mathrm{x}$ is successfully clustered into the first set. However, none of the components of the faults $f_{5}, f_{7}$ and $f_{8}$ can be clustered in the existing sets and hence their clustering is delayed. Next, clustering is attempted for faults with detection count $=3$, that is, $f_{6}$. While neither the first nor the second components of $f_{6}$ can be clustered into the existing sets, the third component of $f_{6}=00 \times x 11 \times x 0 \times$ is successfully clustered into the first set. Next, the algorithm will randomly fill the merged test vectors of the compatibility sets and will fault-simulate the remaining undetected faults, that is $f_{5}, f_{7}$ and $f_{8}$. We assume in this example that fault $f_{5}$ will be detected by the randomly filled test vectors. Finally, $f_{7}$ and $f_{8}$ will be clustered next. The first component of $f_{7}=1 \times 1 \times 1 \times x 10 \times$ is mapped to a new set. Then, the first component of $f_{8}=\times 1 \times x 1 \times x 001$ is found incompatible with the third set and hence its second component is attempted. The second component of $f_{8}=\operatorname{xx} 11 \times 0101$ is then found compatible with the third set and is clustered with it creating the merged test vector $1 \times 111 \times 0101$, which is randomly filled to create a fully specified test set. Thus, the test set is compacted into the following three test vectors: \{0001110100, 1011011001, $1111100101\}$.

\section{Experimental results}

In order to demonstrate the effectiveness of the proposed FCC test compaction technique, we have performed experiments on a number of the ISCAS85 and full-scanned versions of ISCAS 89 benchmark circuits. The experiments were run on a Pentium Mobile, with $2.0 \mathrm{GHz}$ processor and 1GB DDR2 RAM. We have used test sets generated by HITEC [19], which achieve full coverage of all detectable faults in the circuits. HITEC test sets are used for comparison with the work in $[1,10,16]$. In addition, we have used the fault simulator HOPE [20] for fault-simulation purposes and the test relaxation algorithm in [10] for test vector component generation.

In Table 3, we compare the test compaction results of IFC $[1,16]$ and FCC algorithms when applied on the original test set. The first column gives the circuit name. The second column specifies the number of test vectors in the original test set before applying any compaction. The third and fourth columns give test set sizes after applying reverse-order fault simulation (ROF) and random merging (RM) [10], respectively. ROF is based on applying reverse-order and random order fault simulation for 20 iterations. RM is based on relaxing the test vectors generated by ROF and merging compatible vectors. Columns five and six give the results of the IFC technique $[1,16]$ while columns seven and eight report the results of the proposed FCC technique. Test set sizes are given under the column headed \#TV. The CPU time, in seconds, required by each of the techniques is given under the column headed Time. The FCC technique has shown better compaction quality on 12 out of 15 circuits, while two circuits resulted in a draw. In terms of overall savings, FCC has saved more than 120 test vectors than IFC [1, 16] (with an average compaction improvement of 7\%). For example, for the circuits c3540 and c5315, FCC achieved $24 \%$ and $25 \%$ higher compaction than IFC, respectively. Furthermore, FCC consumes significantly lesser CPU time than IFC $[1,16]$. It has shown 13.37 times overall improvement than IFC $[1,16]$.

In order to increase the level of compaction, FCC can be applied in an iterative manner until no compaction improvement is possible. We have experimented with an iterative version of FCC, called FCC6+, by applying FCC iteratively until the length of the test set cannot be reduced in the last six iterations. Unspecified bits in the test set $T$ are assigned random values before every call to the FCC algorithm. Columns nine and ten in Table 3 report the results of an iterative version of IFC applied on the test set generated by ROF, called ROF + ITER_IFC [1, 16]. Columns 11 and 12 report the results of FCC6+. It can be seen that FCC6 + has achieved higher test compaction than ROF + ITER_IFC on 12 out of 15 circuits, while two resulted in a draw. For example, for the circuit c5315, FCC6+ has achieved $29 \%$ more compaction than ROF + ITER_IFC. Furthermore, it has shown higher overall savings (with an average compaction improvement of $8 \%$ ) in a much more efficient CPU time (ranging from 1 to 14 times less CPU time). It should be observed that

Table 2: Illustration of steps of FCC algorithm on the given example

\begin{tabular}{|c|c|c|c|c|c|c|c|c|}
\hline \multirow[b]{2}{*}{ Cluster } & \multicolumn{2}{|c|}{$\begin{array}{l}\text { After mapping faults with } \\
\text { detection count }=1\end{array}$} & \multicolumn{2}{|c|}{$\begin{array}{l}\text { After mapping faults with } \\
\text { detection count }=2\end{array}$} & \multicolumn{2}{|c|}{$\begin{array}{l}\text { After mapping faults with } \\
\text { detection count }=3\end{array}$} & \multirow{2}{*}{$\begin{array}{l}\text { After merging } \\
\text { components } \\
\text { Test vector }\end{array}$} & \multirow{2}{*}{$\begin{array}{l}\text { After random } \\
\text { filling } \\
\text { Test vector }\end{array}$} \\
\hline & Fault & Fault component & Fault & Fault component & Fault & Fault component & & \\
\hline \multirow[t]{3}{*}{1} & $f_{1}$ & $0 \times 0 \times x 1 \times x \times 0$ & $f_{1}$ & $0 \times 0 \times x 1 \times x \times 0$ & $f_{1}$ & $0 \times 0 \times x 1 \times x \times 0$ & $000 \times 11 \times 100$ & 0001110100 \\
\hline & & & $f_{4}$ & $00 x \times 1 \times x 1 x x$ & $f_{4}$ & $00 x \times 1 \times x 1 \times x$ & & \\
\hline & & & & & $f_{6}$ & $00 x \times 11 \times x 0 x$ & & \\
\hline \multirow[t]{2}{*}{2} & $f_{2}$ & $1 \times x 1 \times x 10 \times 1$ & $f_{2}$ & $1 \times x 1 \times x 10 \times 1$ & $f_{2}$ & $1 \times x 1 \times x 10 \times 1$ & $10 \times 1 \times 110 \times 1$ & 1011011001 \\
\hline & & & $f_{3}$ & $x 0 \times 1 \times 1 \times x \times 1$ & $f_{3}$ & $x 0 \times 1 \times 1 \times x \times 1$ & & \\
\hline
\end{tabular}


Table 3: Comparison of compaction results

\begin{tabular}{|c|c|c|c|c|c|c|c|c|c|c|c|}
\hline \multirow[t]{2}{*}{ Circuit } & \multirow[t]{2}{*}{ Orig. \#TV } & \multirow{2}{*}{$\begin{array}{l}\text { ROF } \\
\text { \#TV }\end{array}$} & \multirow{2}{*}{$\begin{array}{l}\text { RM[10] } \\
\text { \#TV }\end{array}$} & \multicolumn{2}{|c|}{$\mathrm{IFC}[1,16]$} & \multicolumn{2}{|l|}{ FCC } & \multicolumn{2}{|c|}{$\begin{array}{l}\text { ROF + IFC- } \\
\text { ITR }[1,16]\end{array}$} & \multicolumn{2}{|c|}{ FCC6+ } \\
\hline & & & & \#TV & Time(s) & \#TV & Time(s) & \#TV & Time(s) & \#TV & Time(s) \\
\hline c2670 & 154 & 106 & 100 & 98 & 0.993 & 98 & 0.04 & 85 & 42.07 & 82 & 3.93 \\
\hline c3540 & 350 & 83 & 80 & 99 & 2.01 & 75 & 1.01 & 75 & 26.95 & 63 & 5.05 \\
\hline c5315 & 193 & 119 & 106 & 107 & 3.97 & 80 & 1.96 & 86 & 88.04 & 61 & 10.94 \\
\hline $\mathrm{s} 13207.1 \mathrm{f}$ & 633 & 476 & 252 & 244 & 34.06 & 238 & 10.02 & 238 & 473.12 & 234 & 69.02 \\
\hline s15850.1f & 657 & 456 & 181 & 142 & 50.97 & 144 & 15.97 & 129 & 374.95 & 118 & 1365.98 \\
\hline s208.1f & 78 & 33 & 33 & 34 & 0.001 & 32 & 0.001 & 32 & 0.01 & 32 & 0.01 \\
\hline s3271f & 256 & 115 & 76 & 60 & 1.95 & 59 & 1.93 & 60 & 18.98 & 55 & 3.95 \\
\hline s3330f & 704 & 277 & 248 & 238 & 3.05 & 230 & 0.99 & 196 & 30.02 & 192 & 4.2 \\
\hline s3384f & 240 & 82 & 75 & 72 & 1.98 & 72 & 0.96 & 72 & 7.07 & 72 & 2.98 \\
\hline s38417f & 1472 & 822 & 187 & 150 & 838 & 130 & 225.95 & 120 & 3775.06 & 108 & 2337 \\
\hline s38584f & 1174 & 819 & 232 & 148 & 4718 & 138 & 154.02 & 124 & 8217.08 & 114 & 1735.17 \\
\hline s4863f & 132 & 65 & 59 & 50 & 3.02 & 47 & 3.95 & 42 & 70.88 & 38 & 6.96 \\
\hline s5378f & 359 & 252 & 145 & 120 & 3.05 & 119 & 1 & 117 & 109 & 107 & 13.99 \\
\hline$s 6669 f$ & 138 & 52 & 42 & 40 & 7.91 & 36 & 5.02 & 30 & 175.01 & 28 & 12.02 \\
\hline s9234.1f & 620 & 375 & 202 & 182 & 11.06 & 170 & 3.04 & 155 & 200.93 & 139 & 27.04 \\
\hline
\end{tabular}

Table 4: Comparison with Mintest [17] dynamic and static compaction test sets

\begin{tabular}{lllllr}
\hline Circuit & FCC6+ & & $\begin{array}{l}\text { Mintest } \\
\text { dynamic [15] }\end{array}$ & \multicolumn{2}{c}{ Mintest static [15] } \\
\hline s5378f & \#TV & CPU time & \#TV & \#TV & CPU time \\
s9234.1f & 107 & 13.99 & 111 & 97 & 131.5 \\
s13207.1f & 139 & 27.04 & 159 & 105 & 3157.1 \\
s15850.1f & 234 & 69.02 & 236 & 233 & 1178.4 \\
s38417f & 118 & 1365.98 & 126 & 94 & 9252.1 \\
s38584f & 108 & 2337 & 99 & 68 & 28955.8 \\
\hline
\end{tabular}

FCC6+ consumes more time on s15850 at the expense of more compaction as the algorithm continued on iterating due to more compaction improvements achieved.

In Table 4, a comparison is made for the largest circuits between the number of compacted test sets obtained by FCC6+ and those obtained by Mintest [15] using both dynamic and static compaction techniques. It should be observed that Mintest static compaction has reported the smallest known test sizes for several circuits. For five out of the six compared circuits, the test size of FCC6+ is smaller than Mintest dynamic test compaction. Comparison in terms of CPU time is not made as the CPU time taken by Mintest dynamic compaction is not available. However, it is known that running ATPG with dynamic test compaction is slower than regular ATPG mode. While for all the circuits, the number of test vectors obtained by Mintest static compaction is smaller, the CPU time is significantly higher than FCC6+, limiting the practicality of the technique for large industrial circuits.

It should be pointed out that any static compaction algorithm can be used after the proposed FCC algorithm. In fact, given a test set T, the FCC algorithm will generate a new test set $T^{*}$ whose characteristics are different from the characteristics of $T$. Thus, a static compaction algorithm that cannot compact $T$ may manage to compact $T^{*}$.

\section{Conclusions}

In this work, we have proposed a new test compaction technique for combinational circuits based on test vector decomposition and clustering. Test vectors are decomposed and clustered for faults in an increasing order of fault detection count. Experimental results have demonstrated the effectiveness of the proposed technique in achieving higher level of compaction in a much more efficient CPU time than previously proposed clustering-based test compaction techniques. An iterative application of the proposed technique has also shown significant increase in the achieved level of test compaction.

\section{Acknowledgment}

This work is supported by King Fahd University of Petroleum and Minerals under project\#FT-2004/07.

\section{References}

1 El-Maleh, A.H., and Osais, Y.E.: 'Test vector decomposition based static compaction algorithms for combinational circuits', $A C M$ Trans. Design Automat. Electron. Syst., 2003, 8, (4), pp. 430-459

2 Flores, P.F., Neto, H.C., and Marques-Silva, J.P.: 'On applying set covering models to test set compaction'. Proc. 9th Great Lakes Symposium on VLSI, Ypsilanti, Michigan, March 1999, pp. 8-11 
3 Boateng, K.O., Konishi, H., and Nakata, T.: 'A method of static compaction of test stimuli'. Proc. Asian Test Symp., Kyoto, Japan, November 2001, pp. 137-142

4 Hochbaum, D.S.: 'An optimal test compression procedure for combinational circuits', IEEE Trans. Comput. Aided Design Integr Circuits Syst., 1996, 15, (10), pp. 1294-1299

5 Schulz, M.H., Trischler, E., and Sarfert, T.M.: 'SOCRATES: a highly efficient automatic test pattern generation system', IEEE Trans. Comput. Aided Design Integr. Circuits Systems, 1998, 7, (1), pp. $126-137$

6 Pomeranz, I., and Reddy, S.M.: 'Forward-looking fault simulation for improved static compaction', IEEE Trans. Comput. Aided Design Integr. Circuits Syst., 2001, 20, (10), pp. 1262-1265

7 Hamzaoglu, I., and Patel, J.H.: 'Test set compaction algorithms for combinational circuits'. Proc. Int. Conf. on Computer-Aided Design, San Jose, CA, November 1998, pp. 283-289

8 Kajihara, S., Pomeranz, I., Kinoshita, K., and Reddy, S.M.: 'Cost effective generation of minimal test sets for stuck-at faults in combinational logic circuits', IEEE Trans. Comput. Aided Design Integr. Circuits Syst., 1995, 14, (12), pp. 1496-1504

9 Lin, X., Rajski, J., Pomeranz, I., and Reddy, S.M.: 'On static test compaction and test pattern ordering for scan designs'. Proc. Int. Test Conf., Washington, DC, USA, 2001, pp. 1088-1098

10 El-Maleh, A., and Al-Suwaiyan, A.: 'An efficient test relaxation technique for combinational and full-scan sequential circuits'. Proc. of VLSI Test Symposium, Monterey, CA, 2002, pp. 53-59

11 Ayari, B., and Kaminska, B.: 'A new dynamic test vector compaction for automatic test pattern generation', IEEE Trans. Comput. Aided Design Integr. Circuits Syst., 1994, 13, (3), pp. 353-358
12 Miyase, K., Kajihara, S., and Reddy, S.M.: 'A method of static test compaction based on don't care identification'. Proc. First IEEE Int. Workshop on Electronic Design, Test, and Application, Christchurch, New Zealand, January 2002, pp. $392-395$

13 Chang, J.-S., and Lin, C.-S.: 'Test set compaction for combinational circuits', IEEE Trans Comput. Aided Design Integr. Circuits Syst., 1995, 14, (11), pp. 1370-1378

14 Reddy, L.N., Pomeranz, I., and Reddy, S.M.: 'ROTCO: a reverse order test compaction technique'. Proc. EURO-ASIC Conf., Paris, France, June 1992, pp. 189-194

15 Hamzaoglu, I., and Patel, J.H.: 'Test set compaction algorithms for combinational circuits', IEEE Trans Comput. Aided Design Integr. Circuits Syst., 2000, 19, (8), pp. 957-963

16 Osais, Y.E., and El-Maleh, A.H.: 'A static test compaction technique for combinational circuits based on independent fault clustering'. Proc. 10th IEEE Int. Conf. on Electronics, Circuits and Systems, Sharga, United Arab Emirates, December 2003, vol. 3, pp. 1316-1319

17 El-Maleh, A.H., and Osais, Y.E.: 'A class-based clustering static compaction technique for combinational circuits'. Proc. 16th Int. Conf. on Microelectronics, Tunis, Tunisia, December 2004, pp. 522-525

18 Kajihara, S., Pomranz, I., Kinoshita, K., and Reddy, S.M.: 'On compacting test sets by addition and removal of test vectors'. VLSI Test Symp., Cherry Hill, USA, April 1994, pp. 25-28

19 Niermann, T.M., and Patel, J.H.: 'HITEC: a test generation package for sequential circuits'. Proc. European Conf. Design Automation, Amsterdam, The Netherlands, 1991, pp. 214-218

20 Lee, H.K., and Ha, D.S.: 'HOPE: an efficient parallel fault simulator for synchronous sequential circuits', IEEE Trans. Comput. Aided Design Integr. Circuits Syst., 1996, 15, (9), pp. 1048-1058 